\title{
Naming and locating simultaneously and sequentially presented letters
}

\author{
CAROL L. KRUMHANSL \\ Stanford University, Stanford, California 94305
}

\begin{abstract}
Two letters varying in level of confusability were presented either simultaneously for $75 \mathrm{msec}$ or sequentially for $75 \mathrm{msec}$ each in adjacent retinal locations. The retinal locus of presentation varied from trial to trial, and subjects both identified and located the presented letters. Identification accuracy was higher on nonconfusable than on confusable letter pairs in the simultaneous condition, but not in the sequential condition. This result is interpreted as support for the notion that inhibition between similar or identical features shared by confusable letters occurs only when letters are presented simultaneously. A relative position effect, with performance on the peripheral letter higher than on the central letter, was found for simultaneously and second sequentially presented letters, but not for first sequentially presented letters. This result is interpreted in terms of the assumption that feature perturbations, with foveal perturbations more likely than peripheral perturbations, affect simultaneously and secondpresented letters, but not first-presented letters. The pattern of results for relative location accuracy showed many of the same effects as identification performance. A model assuming location errors reflect feature transpositions is outlined and is able to account for the absolute and relative location results and the correlation between relative location and identification accuracy.
\end{abstract}

The aim of the present study is twofold. First, the study constitutes an attempt to determine the time course of two mechanisms thought to be involved in the letter identification process, inhibition between input channels at the feature extraction stage (Estes, 1972; Krumhansl \& Thomas, 1977) and perturbation of extracted features in the sensory store which affects the letter identification stage (Wolford, 1975). The second purpose was to further investigate the role of location information in the letter identification process (Estes, Allmeyer, \& Reder, 1976; Krumhansl \& Thomas, 1976).

The notion of inhibition between input channels was originally suggested by Estes (1972) in his interactive channels model. According to his formulation, excitation of an input channel going to a feature detector exerts an inhibitory effect on nearby input channels going to either the same feature detector or other feature detectors. If inhibition is assumed to primarily affect input channels going to the same feature detector (Krumhansl \& Thomas, 1977), then, due to inhibition, greater competition for access to feature detectors will occur when confusable

The work was supported by a National Institutes of Health training grant (MH-10478-09) to the author and a National Science Foundation grant (GB-43275) to Ewart A. C. Thomas. The helpful comments of Ewart A. C. Thomas, Edward E. Smith, and three reviewers on an earlier version of the paper are gratefully acknowledged. Reprint requests may be sent to Carol L. Krumhansl, Department of Psychology, Stanford University, Stanford, California 94305. letters, which share features, are presented. This would result in the extraction of fewer features overall from confusable letter arrays than from nonconfusable letter arrays. Thus, lower identification performance would be expected for confusable letter arrays. This prediction was tested in a report paradigm (Krumhansl \& Thomas, 1977) in which two letters were presented on each trial for identification. The letters were either similar or dissimilar in terms of visual features and, as predicted, identification accuracy was found to be lower for confusable letter pairs than for nonconfusable letter pairs.

The possibility that feature perturbations may occur in the sensory store was originally proposed by Wolford (1975) in his feature perturbation model. This model assumes that extracted letter features are arranged in feature groups in the sensory store corresponding to the letter locations in the display. Over time, perturbations of features are assumed to occur between the feature groups. In addition, it is assumed that the probability of a feature perturbation increases with increased distance into the periphery, that perturbations in a central direction are more probable than perturbations in a peripheral direction, and that feature perturbations are detrimental to letter identification, since after peturbations have occurred the features contained in a feature group may no longer be compatible, that is, could not possibly have been extracted from any one letter. The model was used by Wolford to account for a number of effects such as relative position, string position, retinal location, and report order 
found in report experiments. Of particular interest for the present investigation is the relative position effect found in a number of studies (cf. Bouma, 1970, 1973; Krumhansl \& Thomas, 1977; Shaw, 1969; Wolford \& Hollingsworth, 1974). In these studies, performance at a fixed retinal location was impaired more if there was a neighboring letter on its peripheral side than if there was a neighboring letter on its central side. Wolford's model is able to account for this effect in terms of the assumption that central perturbations are more probable than peripheral perturbations, so that a letter location is more subject to detrimental feature perturbations from a peripheral than from a central neighboring letter.

Although not explicitly considered by Wolford (1975), the feature perturbation model seems to make at least two predictions concerning the effect of level of confusability between presented letters. First, feature perturbations might be expected to be less detrimental for confusable than for nonconfusable letters. This would be true since the arrival of a feature into a feature group would not be expected to interfere with, and may even facilitate, identification if the arriving feature is compatible with the other features in the group. Since confusable letters have more features in common, and hence more mutually compatible features, performance should be better for confusable than for nonconfusable letters in the absence of inhibition. Second, since the relative position effect is assumed to result from the greater probability of foveal perturbations than peripheral perturbations and these perturbations would seem to be more detrimental for nonconfusable letters, the magnitude of the relative position effect should be larger for nonconfusable than for confusable letters. While the first predicted result was not obtained in the earlier study (Krumhansl \& Thomas, 1977), the predicted interaction between level of confusability and relative position was found, as was an overall relative position effect. The results of the earlier study were interpreted in terms of a model incorporating both feature specific inhibition and feature perturbation assumptions.

In an attempt to determine the time course of inhibition and feature perturbations, two letters were presented either simultaneously or sequentially in the present study. As in the earlier study, the two letters were either confusable or nonconfusable. A number of predictions can be made for sequentially presented letters. Considering first the effect of inhibition, it might be supposed that features of the first of sequentially presented letters would be extracted before inhibition from the second letter occurs. First-presented letters, then, would show no effect of the level of confusability between the two letters on the trial. For second-presented letters, inhibition from the first letter may affect extraction of features from the second letter, but if the strength of inhibition decreases over time the effect of inhibition would be less than for simultaneously presented letters. Thus, a smaller effect of level of confusability might be expected for second-presented letters than for simultaneously presented letters, and no effect of level of confusability would be expected for first-presented letters. Considering now the effect of feature perturbations on the identification of sequentially presented letters, if the first letter is identified before perturbations from the second letter occur, then first-presented letters should show no relative position effect and no interaction between level of confusability and relative position. For second-presented letters, however, if the letters are presented in close enough temporal succession, features of both letters may be simultaneously present in the sensory store and perturbations from the first letter may affect identification of the second letter. Assuming that perturbations are biased in a central direction, this would predict a relative position effect and an interaction between level of confusability and relative position for second-presented letters. And, finally, if the effect of inhibition is negligible for second-presented letters, the feature perturbation model would predict an advantage for confusable second-presented letters over nonconfusable second-presented letters. These predictions are tested in the present experiment:

The second purpose of this study was to further investigate the role of location information in the letter identification process. Two recent studies (Estes et al., 1976; Krumhansl \& Thomas, 1976) have investigated letter location performance in report experiments. In these studies, subjects are asked to both identify and indicate the location of the presented letters. In both studies, location accuracy was found to decline with distance from the center of the visual field, and transposition errors occurred primarily in a central, rather than in a peripheral, direction. In addition, location errors were found to occur more frequently at interior string positions than at end positions, and in the Estes et al. study location accuracy was found to be largely independent of stimulus duration. In the Krumhansl and Thomas study, the probability of designating the correct absolute location of a single presented letter was found to decline more rapidly with increased distance from the center of the visual field than the probability of correctly identifying the letter. The results of this study were interpreted in terms of a model which assumed that identification and location of letters are independent processes. In a more recent study (Krumhansl \& Thomas, 1977), however, inversion errors occurred more frequently on trials with fewer correct identifications, and this 
result is consistent with the finding that transposition errors tended to terminate in the positions of letters which were not correctly reported on the trial in the Estes et al. study. Also in the Krumhansl and Thomas (1977) study, order inversion errors occurred more often when confusable letters were presented, and as in the Estes et al. study, the probability of order inversion was found to be independent of stimulus duration. These studies suggest that the role of location information in letter identification may be different when single letters are presented than when multiple letters are presented. In the multiple-letter situation, if location errors reflect feature transpositions, then poor location performance would be expected to be correlated with poor identification performance. With single letters, however, feature transpositions might not be detrimental, since no incompatible features are present, and hence identification performance may be independent of location performance. These studies also suggest the desirability of distinguishing between the ability to locate letters in the correct absolute location in the visual field and the ability to locate letters in the correct order or relative location, since errors of the first kind might be expected to be less related to identification errors than errors of the second kind.

It is hoped that the results of the present experiment will bear on the question of the interrelationship between the location and identification processes. Under the assumption that order inversions reflect feature perturbations between feature groups, which are detrimental to letter identification, then those conditions with the greatest number of order inversions would be expected in general to also show the poorest identification performance. Further, if feature perturbations are assumed to occur over time and to be biased in a central direction, then a difference might be expected between sequential presentation when the central letter is presented first and sequential presentation when the peripheral letter is presented first. Also, as suggested in an earlier paper (Krumhansl \& Thomas, 1977), since it is assumed that confusable letters share more features, hence have fewer distinctive features, order inversions can occur when fewer feature transpositions have occurred, thus predicting more inversion errors for confusable than for nonconfusable letters.

Finally, with regard to absolute location performance, if feature transpositions occur over time and a decision as to the absolute location of the letters is based on feature locations after all perturbations have occurred, then, since the total duration of the stimulus is longer in the sequential condition, more incorrect absolute location judgments might be expected under sequential than under simul- taneous presentation. If foveal feature transpositions are more likely than peripheral transpositions, then these absolute location errors would be expected to show a foveal bias. The results of the present experiment will be used to test these predictions concerning the effect of simultaneous and sequential presentation on relative and absolute location performance.

\section{METHOD}

\section{Subjects}

The 15 Stanford University students who served as subjects received credit towards introductory psychology. Subjects had normal or corrected-to-normal vision and participated in two test sessions which lasted approximately $55 \mathrm{~min}$ each.

\section{Apparatus}

The experiment was run using a programmable CRT display (Imlac Corporation PDS-1) interacting with a PDP-10 timesharing computer system. The test display appeared on the screen in green characters against a dark gray background and a headrest was used to maintain a viewing distance of $43 \mathrm{~cm}$. Subjects wrote their responses on a separate response form.

\section{Stimulus Materials}

The premask and postmask arrays consisted of 14 noise characters (dollar signs) in a horizontal string with a small $x$ in the center of the array. The array subtended a horizontal visual angle of $10.80^{\circ}$ and a vertical angle of $.79^{\circ}$. The noise characters and letters measured $.53^{\circ}$ wide and $.79^{\circ}$ high. The central $x$ measured $.53^{\circ}$ wide and $.53^{\circ}$ high. Intercharacter spaces were $.26^{\circ}$, except that the $\mathrm{x}$ was immediately adjacent to its flanking noise characters. The response frame which appeared after the postmask was identical to the pre- and postmasks, except that the five central mask characters in each half of the mask array were replaced by dashes, indicating the 10 possible letter positions. On each trial, two letters appeared in two adjacent positions in either the left visual field or the right visual field. Nonletter positions contained unchanging mask characters. On all trials, the most peripheral and the most central position in each visual field was occupied by an unchanging noise character. Confusable trials consisted of two letters both drawn without replacement from one of the three sets $[B, D, P, R],[F, H, L, T]$, or $[M, N$, $\mathrm{V}, \mathrm{W}]$. Nonconfusable trials consisted of two letters drawn from different sets. The choice of these sets of confusable letters is based on the observation that average intragroup confusability is larger than average intergroup confusability in Townsend's (1971) confusion data. Across trials, each letter appeared equally often on nonconfusable and confusable trials. Fifteen blocks of 32 trials each were constructed. Five blocks consisted of simultaneous trials and 10 blocks consisted of sequential trials.

Simultaneous trials. On simultaneous trials, two letters simultaneously replaced two adjacent mask characters for $75 \mathrm{msec}$. Sixteen simultaneous trial types were defined by level of confusability (confusable or nonconfusable), visual field (left or right), and retinal locus of presentation (Retinal Locations 1 and 2, 2 and 3,3 and 4 , or 4 and 5 , were retinal locations are numbered as in Table 1). Each simultaneous trial type appeared twice in each of the five blocks of simultaneous trials, so that across blocks 10 observations for each trial type were made. Table 1 shows a sample simultaneous trial.

Sequential trials. On sequential trials, one letter replaced a noise character for $75 \mathrm{msec}$. Simultaneously with the offset of the first target letter, a second letter replaced an adjacent noise character for $75 \mathrm{msec}$. Thirty-two trial types were defined by levels of confusability (confusable or nonconfusable), visual field (left or right), retinal locus of presentation (Retinal Locations 1 and 2, 2 and 3,3 and 4 , or 4 and 5), and order of presentation (central- 
Table 1

Sample Simultaneous and Sequential Trials

\begin{tabular}{|c|c|c|c|c|c|c|c|c|c|c|c|c|c|c|c|c|}
\hline \multirow[b]{2}{*}{ Trial Type } & \multirow[b]{2}{*}{ Frame Type } & \multicolumn{15}{|c|}{ Retinal Location } \\
\hline & & & 5 & 4 & 3 & 2 & 1 & & & & 1 & 2 & 3 & 4 & 5 & \\
\hline & Premask & $\$$ & $\$$ & $\$$ & $\$$ & $\$$ & $\$$ & $\$$ & $\mathrm{x}$ & $\$$ & $\$$ & $\$$ & $\$$ & $\$$ & $\$$ & $\$$ \\
\hline Simultaneous & Stimulus ( $75 \mathrm{msec})$ & $\$$ & $\$$ & $\mathrm{~L}$ & $\mathrm{~T}$ & $\$$ & $\$$ & $\$$ & $\mathbf{x}$ & $\$$ & $\$$ & $\$$ & $\$$ & $\$$ & $\$$ & $\$$ \\
\hline \multirow[t]{3}{*}{ Confusable } & Postmask (1 sec) & $\$$ & $\$$ & $\$$ & $\$$ & $\$$ & $\$$ & $\$$ & $\mathrm{x}$ & $\$$ & $\$$ & $\$$ & $\$$ & $\$$ & $\$$ & $\$$ \\
\hline & Response Frame & $\$$ & - & - & - & - & - & $\$$ & $\mathrm{x}$ & $\$$ & - & - & - & - & - & $\$$ \\
\hline & Premask & $\$$ & $\$$ & $\$$ & $\$$ & $\$$ & $\$$ & $\$$ & $\mathrm{x}$ & $\$$ & $\$$ & $\$$ & $\$$ & $\$$ & $\$$ & $\$$ \\
\hline Sequential & Stimulus (75 msec) & $\$$ & $\$$ & $\$$ & $\$$ & $\$$ & $\$$ & $\$$ & $\mathrm{x}$ & $\$$ & $\$$ & $\$$ & $\$$ & $\$$ & $\mathbf{P}$ & $\$$ \\
\hline (Peripheral-Central) & Stimulus (75 msec) & $\$$ & $\$$ & $\$$ & $\$$ & $\$$ & $\$$ & $\$$ & $\mathrm{x}$ & $\$$ & $\$$ & $\$$ & $\$$ & V & $\$$ & $\$$ \\
\hline \multirow{2}{*}{ Nonconfusable } & Postmask (1 sec) & $\$$ & $\$$ & $\$$ & $\$$ & $\$$ & $\$$ & $\$$ & $\mathrm{x}$ & $\$$ & $\$$ & $\$$ & $\$$ & $\$$ & $\$$ & $\$$ \\
\hline & Response Frame & $\$$ & _- & - & - & - & - & $\$$ & $x$ & $\$$ & - & - & - & - & - & $\$$ \\
\hline
\end{tabular}

peripheral or peripheral-central). Each trial type appeared once in each of the 10 blocks of sequential trials, so that across blocks 10 observations for each trial type were made. Table 1 shows a sample sequential trial.

\section{Procedure}

At the start of the first test session, subjects were told that on each trial the following sequence of events would occur: (a) The premask array would appear centered on the screen. (b) After the subject had fixated on the $\mathrm{x}$ in the center of the array, the subject would press the spacebar on the typewriter-like keyboard in front of the CRT screen. (c) Two letters would briefly displace noise characters, appearing either simultaneously or sequentially, but always in adjacent positions and never at either the most peripheral or the most central position in either visual field. (d) The postmask array would then appear and remain on the screen for $1 \mathrm{sec}$. (e) Finally, the response frame would appear, indicating the five possible letter positions in each visual field, and would remain on the screen until the subject had written his response and initiated the next trial. The subjects were instructed to indicate what letters they saw and where they saw them by writing the letters in the corresponding letter positions on the response form. The subjects were encouraged to guess as to the identity of a letter on the basis of partial information, but to always indicate which positions contained letters by writing an $\mathrm{x}$ in the position when they were unable to identify a letter.

At the start of the first test session, eight simultaneous and eight sequential practice trials were followed by seven blocks of trials, so that the eight remaining blocks of trials were presented in the second test session which was scheduled on a different day. For each subject, trials were randomly ordered within the blocks, and the blocks were randomly ordered subject to the constraint that, within each group of three blocks, one block consist of simultaneous trials and two blocks consist of sequential trials. At the start of each block of trials, a message indicated to the subject whether the trials to be presented in that block would be simultaneous or sequential.

\section{RESULTS}

\section{Identification Performance}

The average probability of correct identification is shown in Figure 1 as a function of mode of presentation (simultaneous, peripheral-central sequential, and central-peripheral sequential), level of confusability (confusable or nonconfusable), retinal locus of presentation (Retinal Locations 1 and 2, 2 and 3, 3 and 4 , or 4 and 5), and relative position (central or peripheral). These points represent the probability that the letter presented on a trial is contained some- where in the subject's response, not necessarily in the correct location. The values of the points plotted are contained in Table 2. The analysis of variance on the identification data used as scores for each subject the probability of correct identification at each of the two letter locations for each trial type. The five factors in the analysis were mode of presentation, locus of presentation, level of confusability, relative position, and visual field.

Overall, identification accuracy was highest in the central-peripheral sequential condition (.573), intermediate in the simultaneous condition (.545), and lowest in the peripheral-central sequential condition (.511), yielding an $F(2,28)=5.426, p<.05$. Performance in the simultaneous condition was no different from performance averaged over the two sequential conditions. Identification accuracy decreased with increased distance from the fixation point, $F(3,42)=55.476, p<.01$. This effect of locus of presentation was smallest in the centralperipheral condition, intermediate in the simultaneous condition, and largest in the peripheralcentral condition; this interaction resulted in an $\mathrm{F}(6,84)=2.409, \mathrm{p}<.05$.

Level of confusability interacted with mode of presentation, $F(2,28)=14.258, p<.01$. In the simultaneous condition, average performance on nonconfusable trials (.578) was higher than on confusable trials (.511), whereas for the sequential conditions there was a slight advantage for confusable trials (.546) over nonconfusable trials (.538). In addition, the interaction between level of confusability, mode of presentation, and relative position was significant, $F(2,28)=7.134, p<.01$. Direct comparisons can be made at Retinal Locations 2, 3 , and 4. For simultaneous letters, there was a larger difference at these retinal locations between nonconfusable and confusable trials if that retinal location contained the more peripheral letter on the trial than if it contained the more central letter on the trial. The average difference between nonconfusable and confusable identification accuracy at these retinal locations was .134 for letters in the peripheral relative position and .013 for letters in the central 


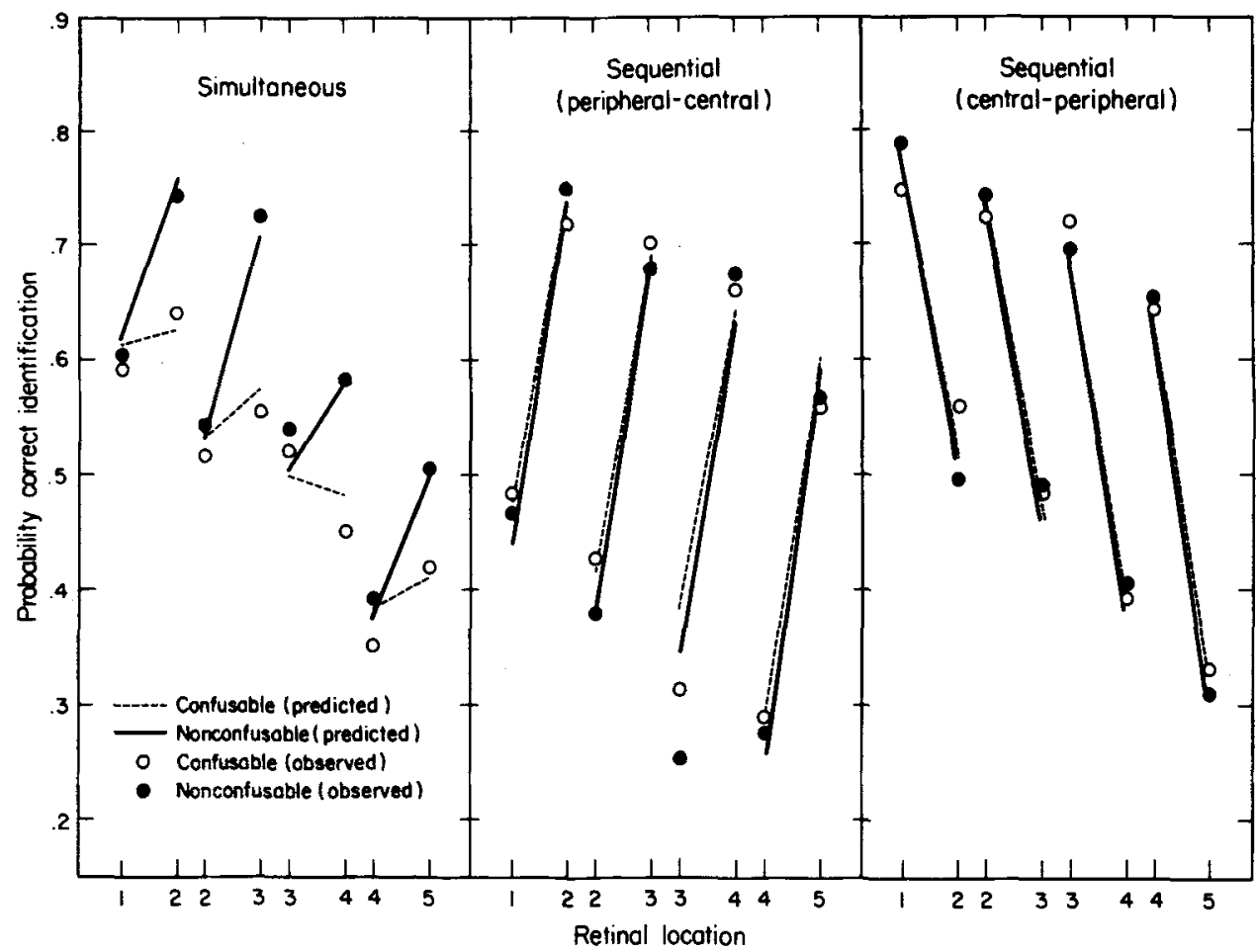

Figure 1. Prohability of correct letter identification in the simultaneous, the peripheral-central sequential, and the central-peripheral sequential conditions. The estimated and predicted values for nonconfusable and confusable letter pairs are shown at each retinal location of presentation. The lines connect the estimated values for the two letters at each locus of presentation (Retinal Locations 1 and 2 , 2 and 3,3 and 4 , or 4 and 5 ).

relative position. For first sequentially presented letters, there was no difference at these retinal locations between performance on nonconfusable and confusable trials for either central or peripheral letters. The average difference between accuracy on nonconfusable and confusable first-presented letters was -.001 for central letters and .009 for peripheral letters. For second sequentially presented letters there was an advantage for confusable letters which was slightly larger for central letters than for peripheral letters. The average difference between nonconfus- able and confusable second-presented letters at these retinal locations was - .040 for central letters and -.016 for peripheral letters, the negative values indicating an advantage for confusable over nonconfusable trials.

Relative position, that is, whether a letter is in the more central or the more peripheral position on the trial, also interacted with mode of presentation, $F(2,28)=47.074, p<.01$. Again, direct comparisons can be made at Retinal Locations 2, 3 and 4 . For simultaneously presented letters at these retinal

Table 2

Observed Probability of Correct Identification for Confusable and Nonconfusable Letters at Central and Peripheral Relative Positions in the Simultaneous and Sequential Conditions

\begin{tabular}{|c|c|c|c|c|c|c|c|c|c|c|c|c|}
\hline \multirow{3}{*}{$\begin{array}{l}\text { Retinal } \\
\text { Locus }\end{array}$} & \multicolumn{4}{|c|}{ Simultaneous } & \multicolumn{4}{|c|}{ Sequential (P-C) } & \multicolumn{4}{|c|}{ Sequential (C-P) } \\
\hline & \multicolumn{2}{|c|}{ Conf } & \multicolumn{2}{|c|}{ Nonc } & \multicolumn{2}{|c|}{ Conf } & \multicolumn{2}{|c|}{ Nonc } & \multicolumn{2}{|c|}{ Conf } & \multicolumn{2}{|c|}{ Nonc } \\
\hline & $\mathrm{C}$ & $\mathbf{P}$ & $\mathrm{C}$ & $\mathbf{P}$ & $\mathrm{C}$ & $\mathbf{P}$ & $\mathrm{C}$ & $\mathbf{P}$ & $\mathrm{C}$ & $\mathbf{P}$ & $\mathrm{C}$ & $\mathbf{P}$ \\
\hline 1,2 & .590 & .640 & .603 & .740 & .480 & .717 & .470 & .750 & .747 & 563 & .783 & .493 \\
\hline 2,3 & .517 & .553 & .540 & .727 & .427 & .700 & .377 & .677 & .723 & .480 & .743 & .483 \\
\hline 3,4 & .570 & .453 & .540 & .583 & .310 & .660 & .250 & .677 & .723 & .387 & .690 & .407 \\
\hline 4,5 & .347 & .420 & .393 & .500 & .287 & .557 & .277 & .567 & .643 & .337 & .653 & .310 \\
\hline
\end{tabular}

Note-P-C and C-P refer to the peripheral-central and the central-peripheral sequential orders, Conf and Nonc refer to confusable and nonconfusable trials, and $C$ and $P$ refer to the central and peripheral relative positions, respectively. 
locations, average performance for central letters was .484 and average performance for peripheral letters was .616 . For sequentially presented letters at these retinal locations, performance was much higher for first-presented letters than for second-presented letters. For first-presented letters, average identification accuracy at these retinal locations was .696 and .697 for central and peripheral letters, respectively. Thus, for first-presented letters, there was no effect of relative position. For second-presented letters, average identification accuracy was .321 and .469 for central and peripheral letters, respectively; that is, for second-presented letters there was a relative position effect which was similar in magnitude to the relative position effect found for simultaneously presented letters.

Overall, visual field had no effect, but this variable interacted with relative position, $F(1,14)=21.291$, $\mathrm{p}<.01$, with a larger advantage for peripheral letter positions in the left visual field than in the right visual field, indicating a left-to-right bias. Visual field also interacted with mode of presentation, $F(2,28)=4.882, p<.05$, with an advantage for simultaneous trials in the right visual field and an advantage for sequential trials in the left visual field.

\section{Location Performance}

Two kinds of location performance will be discussed, absolute and relative. On each trial, subjects indicated two adjacent positions as letter positions whether or not they were able to identify the letters presented on the trial. Therefore, each response can be scored according to whether the correct positions are chosen as response location; this measure is called absolute location accuracy. In addition, on those trials with one or more correct letter identifications the response can be scored according to whether the correctly identified letter is in the correct relative position within the two designated locations; this measure is called relative location accuracy.

Figure 2 summarizes absolute location performance. Shown for each locus of presentation are the probabilities that each retinal locus was chosen in the response. These data are collapsed across mode of presentation, level of confusability, and visual field. There was a tendency for more location errors to occur with increased distance from the fixation point and for transposition errors to occur in a foveal rather than in a peripheral direction. The majority of peripheral transpositions occurred when the letters were presented in the most foveal retinal locus.

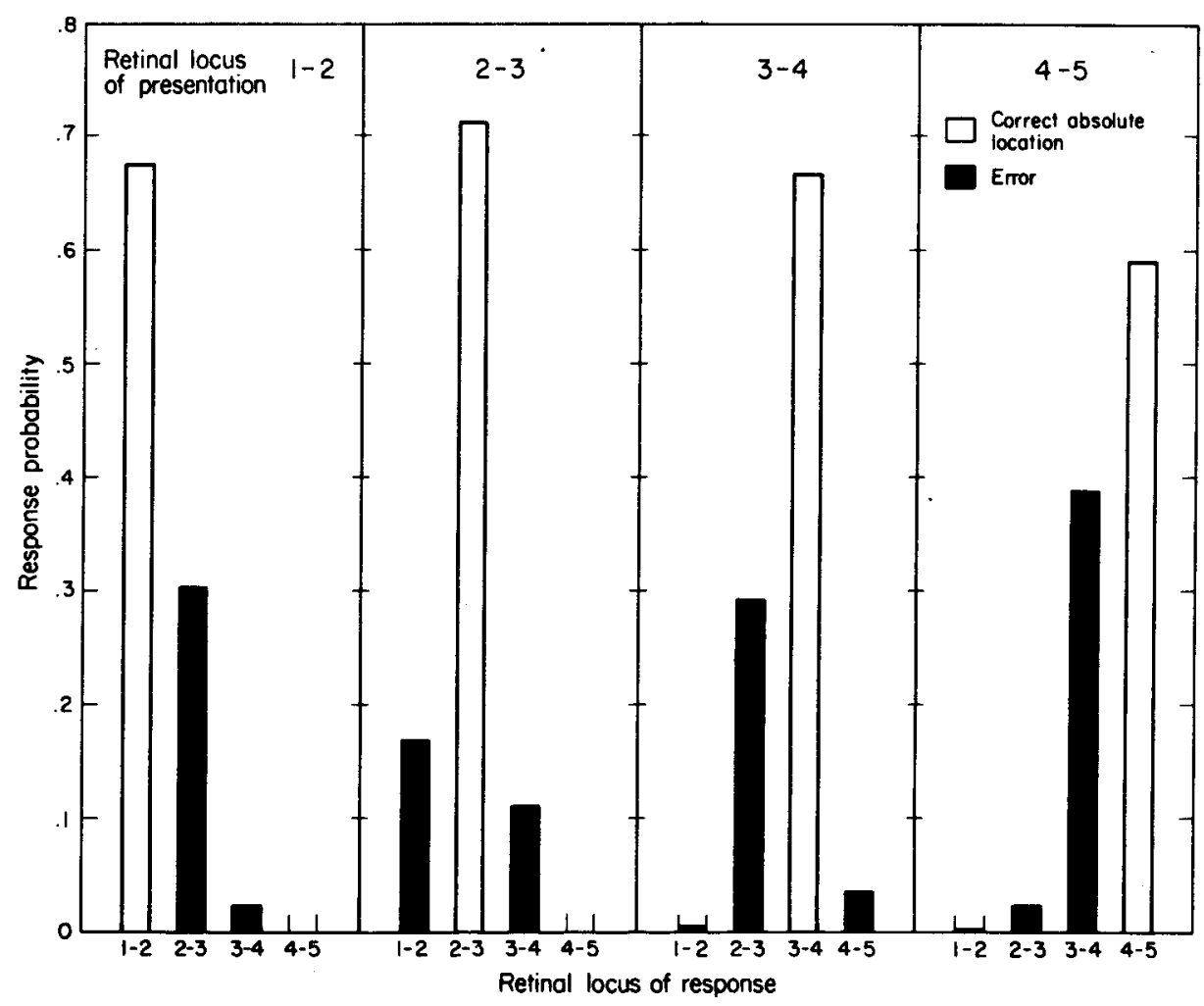

Figure 2. Distribution of absolute location responses for letters presented in each retinal locus (Retinal Locations 1 and 2,2 and 3,3 and 4, and 4 and 5). 
Table 3 summarizes the probability that foveal and peripheral transpositions occurred in the simultaneous, peripheral-central, and central-peripheral sequential conditions. Foveal transpositions occurred slightly more often in the sequential conditions than in the simultaneous condition, although the differences in the probabilities are small. Peripheral transpositions were relatively infrequent and occurred approximately equally often under each mode of presentation. An analysis of variance used as scores for each trial type the probability of correct absolute location for each subject. The analysis showed no effect of mode of presentation, level of confusability, or visual field. Further, the effect of locus of presentation was not significant.

A summary of the relative location data is given in Table 4. Shown are the probabilities of order inversion as a function of level of confusability, locus of presentation, and mode of presentation. An analysis of variance used as scores the probability of order inversion for each subject for each trial type. The probability of order inversion was greater when the letters presented were confusable than when the letters were nonconfusable $[F(1,14)=6.868, p<$ $.05]$, and the probability of order inversion showed an increasing linear trend with distance from the fixation point $[\mathrm{F}(1,42)=5.790, \mathrm{p}<.05]$. Further, the probability of order inversion was highest in the peripheral-central sequential condition, intermediate in the simultaneous condition, and lowest in the central-peripheral sequential condition $[F(2,28)=$ $6.651, \mathrm{p}<.011$. Thus, more inversion errors occurred under the same experimental conditions as fewer correct identifications. This is true even though there are fewer opportunities for an inversion error to be scored when fewer correct identifications are made, since relative location information can only be based on trials with one or two correct identifications.

\section{DISCUSSION}

\section{Identification Performance}

The results of the simultaneous condition of the present study replicated the results of Krumhansl and Thomas (1977). As in the earlier study, subjects identified nonconfusable letter pairs more accurately than confusable letter pairs when the letters were

Table 3

Probabilities of Foveal and Peripheral Absolute Location Errors for Each Mode of Presentation

\begin{tabular}{lccc} 
& \multicolumn{3}{c}{ Probability of Transposition } \\
\cline { 2 - 4 } Condition & Foveal & Peripheral & Total \\
\hline Simultaneous & .271 & .161 & .432 \\
Peripheral-Central & .287 & .165 & .452 \\
Central-Peripheral & .319 & .154 & .473 \\
\hline
\end{tabular}

Table 4

Probability of Order Inversion as a Function of Level of Confusability, Locus of Presentation, and Mode of Presentation

\begin{tabular}{clc}
\hline Variable & \multicolumn{1}{c}{ Condition } & $\begin{array}{c}\text { Probability } \\
\text { of Order } \\
\text { Inversion }\end{array}$ \\
\hline Level of Confusability & Confusable & .216 \\
& Nonconfusable & .181 \\
& Locations 1 and 2 & .173 \\
Locus of Presentation & Locations 2 and 3 & .195 \\
& Locations 3 and 4 & .214 \\
& Locations 4 and 5 & .212 \\
& Simultaneous & .188 \\
Mode of Presentation & Peripheral-Central & .242 \\
& Central-Peripheral & .165 \\
\hline
\end{tabular}

presented simultaneously. This result is consistent with a number of detection experiments (Estes, 1972; Gardner, 1973; McIntyre, Fox, \& Neale, 1970; Shiffrin \& Gardner, 1972), in which it was found that, both in terms of accuracy and latency, target detection is better if target and noise characters are dissimilar than if they are similar. A second finding was that identification of a letter in a retinal location was better if the other simultaneously presented letter occupied a more central, rather than a more peripheral, retinal location. This relative position effect has also been reported in other studies (Bouma, 1970, 1973; Shaw, 1969; Wolford \& Hollingsworth, 1974). Finally, the relative position effect was larger for nonconfusable than for confusable letters, or, put another way, the advantage for nonconfusable over confusable letters was larger for peripheral letters than for central letters.

The results of the earlier study (Krumhansl \& Thomas, 1977) were interpreted in terms of a quantitative model incorporating both feature-specific inhibition and feature-perturbation assumptions. The overall effect of level of confusability was assumed to result from inhibition between similar or identical features when confusable letters are presented. Inhibition has the effect of reducing the number of features available for letter identification and thus lowering identification accuracy. An alternate interpretation of the effect of level of confusability is, however, possible. If the number of input channels to feature detectors is limited, then even if inhibition is not feature-specific, greater competition for access to feature detectors may occur when confusable letters, which share features, are presented in close spatial proximity. This interpretation would seem consistent with Estes' (1972) original formulation in which inhibition was assumed to affect similar and dissimilar feature detectors equally. The relative position effect found in the earlier study was accounted for in terms of the proposed model by the greater probability that feature perturbations occur in a central direction than in a 
peripheral direction. Thus, a letter location would be more subject to detrimental feature perturbations from a peripheral neighboring letter than from a central neighboring letter. Finally, the larger relative position effect found for nonconfusable letters was explained in terms of the greater probability for nonconfusable letters that a feature perturbation, which tends to occur in a central direction, is detrimental to identification. This is true since nonconfusable letters have fewer features in common than confusable letters, and so a feature perturbation would more often result in the combination of incompatible features, that is, distinctive features of two different letters.

Although identification performance averaged over the two sequential conditions was no different from the simultaneous condition in the present experiment, similar to the results of Shiffrin and Gardner (1972), the pattern of results in the sequential conditions differed in a number of respects from the simultaneous condition. First, when a distinction is made between the two sequential conditions in terms of the order of presentation (peripheralcentral or central-peripheral), identification accuracy in all three conditions was not equal. Performance was highest in the central-peripheral sequential condition, intermediate in the simultaneous condition, and lowest in the peripheral-central sequential condition. Further, retinal locus (distance from the fixation point) had the smallest effect in the central-peripheral condition, an intermediate effect in the simultaneous condition, and the largest effect in the peripheralcentral condition.

Difference vere also found in identification accuracy as a sunction of order of presentation. Identification of first-presented letters was better than identification of simultaneously presented letters, and identification performance was lowest for second-presented letters. A similar advantage for first over second sequentially presented letters has been found in a number of other studies (Arabie, 1974; Eriksen \& Eriksen, 1971, Experiment 1; Mayzner \& Greenberg, 1971; Mayzner, Tresselt, Checkes, \& Hoenig, 1970). The advantage for firstpresented letters over simultaneously and secondpresented letters might be accounted for in terms of the number of display positions which are changing simultaneously with letter onset (Estes, Bjork, \& Skaar, 1974; Estes et al., 1976). For first-presented letters, the letter position is the only changing display position; for simultaneously and second-presented letters, a neighboring display position is also changing. However, the difference between simultaneously and second-presented letters cannot be accounted for in terms of the number of simultaneously changing display positions, so it seems preferable to interpret the relatively poor performance on second- presented letters in terms of difficulty in shifting identification processing from the first to the second of sequentially presented letters as suggested by Sperling (1963).

Order of presentation interacted with level of confusability. For simultaneously presented letters, there was an advantage for nonconfusable over confusable letters, for first sequentially presented letters, performance was equal for nonconfusable and confusable letters, and for second sequentially presented letters, there was a slight advantage for confusable letters. The different effect of level of confusability for simultaneous and sequential letters may be related to the finding that the magnitude of the effect of level of confusability for simultaneously presented letters was found to decrease with increased stimulus duration (Krumhansl \& Thomas, 1977). This result of the earlier study was interpreted in terms of the notion that inhibition operates only over a short duration, so that with increased stimulus duration the effect of inhibition between similar features is reduced. Finally, in the present experiment, order of presentation interacted with relative position. Relative position had an effect on second, but not on first, letters and the relative position effect for second letters was similar in magnitude to the relative position effect for simultaneous letters.

The results of the present experiment are interpreted in terms of a model similar to the previously proposed model for letter identification (Krumhansl \& Thomas, 1977). The earlier model is extended to apply to sequentially as well as simultaneously presented letters. Only a qualitative description of the model is given here, but the fit of the model to the data is shown in Figure $1 .^{1}$ In the model it is assumed that both feature-specific inhibition and feature perturbations affect identification of simultaneously presented letters. These mechanisms result in the overall effect of level of confusability, the relative position effect, and the interaction between level of confusability and relative position found for simultaneously presented letters. For first sequentially presented letters, it is assumed that neither inhibition nor perturbations affect performance, that is, identification of the first letter is completed before either inhibition or feature perturbation from the second letter occurs. Thus, for first letters, no effect of level of confusability or relative position is predicted, nor is an interaction between these two variables, consistent with the present results. Finally, for second sequentially presented letters, it is assumed that the effect of inhibition from the first letter is negligible, but that feature perturbations from the first letter may affect identification of the second letter. These perturbations result in the relative position effect found for second-presented letters. Also, since perturbations are less detrimental to identifica- 
tion when letters share features, the model is also able to account for the advantage for confusable over nonconfusable second-presented letters.

In summary, a model assuming that the two mechanisms, feature-specific inhibition and feature perturbation, have different time courses is able to account for the present results. The model assumes that the effective duration of inhibition is short and does not affect identification performance when letters are presented sequentially under the conditions of the present experiment, but that features are maintained in the sensory store for a long enough duration so that perturbation of these features may affect identification of the sequentially presented letters in this experiment.

\section{Location Performance}

The second purpose of the present experiment was to further investigate the role of location information in the letter identification process. With regard to absolute location performance, the majority of transposition errors occurred in a central rather than in a peripheral direction. Absolute location performance was unaffected by level of confusability. In addition, there was only a slight increase in absolute location errors with distance from the center of the visual field and only a small effect of mode of presentation, with slightly more transposition errors occurring in the sequential conditions. Thus, those conditions showing decrements in identification performance were not accompanied by substantial decrements in absolute location accuracy. These results might be considered support for the notion (Krumhansl \& Thomas, 1976) that identifying and locating letters absolutely within the visual field are largely independent processes.

The pattern of results with regard to relative location accuracy was quite different from absolute location accuracy and was similar in many respects to the identification data. The probability of correct relative location was greatest, that is, the number of order inversions was smallest, in the centralperipheral condition, intermediate in the simultaneous condition, and lowest in the peripheralcentral condition, following the same pattern as identification performance. In addition, relative location performance was better on nonconfusable than on confusable trials (Krumhansl \& Thomas, 1977 ) and the probability of correct relative location decreased with increased distance from the center of the visual field.

These results suggest a general model of location performance. ${ }^{2}$ The model assumes, as suggested by Wolford (1975), that feature perturbations tend to occur in a central direction, occur with greater probability at peripheral retinal locations, and occur over time. Under the assumption that absolute location decisions are based on the location of features after perturbations have occured, this model predicts a greater number of foveal than peripheral absolute location errors and an increase in these errors with distance into the periphery. Further, more absolute location errors might be expected to occur with sequential presentation, since more time has elapsed before the location decision is made when letters are presented sequentially. While the results of the present experiment do not provide strong support for these predictions, each of these trends are present in the absolute location data.

This general model for location performance also makes various predictions concerning relative location performance. An effect of mode of presentation on the number of order inversions is predicted. In the sequential condition in which the central letter is presented first, perturbations of these features over time would tend to move them in a central direction, away from the later arriving features of the second, more peripheral letter, thus tending to decrease the spatial overlap of the two feature groups and resulting in relatively few order inversions and greater identification accuracy. When the peripheral letter is presented first, however, perturbations over time would tend to move these features closer to the more central location, thus increasing the spatial overlap of the feature groups and decreasing both relative location and identification accuracy. Simultaneously presented letters would be expected to be associated with an intermediate amount of spatial overlap of the feature groups, and hence intermediate relative location and identification performance. These predictions are confirmed in the present experiment. Further, since more feature perturbations are assumed to occur at peripheral locations, the number of order inversions would be expected to increase with increased distance into the periphery, a result also obtained in the present experiment. It is interesting to note, too, that identification performance declines most rapidly in those conditions with the greatest probability of order inversion, supporting the idea that feature perturbations play a role in the decline in identification accuracy with distance into the periphery when multiple letters are presented. Finally, the observation that inversion errors were more common for confusable than for nonconfusable letters can be explained if it is assumed that order inversions result when the distinctive features from the peripheral letter are contained in a more central location than the distinctive features from the central letter. Since confusable letters have more features in common, and hence fewer distinctive features, an order inversion could result when fewer feature perturbations have occurred, and therefore more order inversions are expected for confusable letters. 
In summary, the results of the present experiment concerning both absolute and relative location performance can be accounted for in terms of a model which incorporates feature perturbation assumptions. This approach suggests that both location and identification errors result in part from the same underlying feature perturbation mechanism. Specifically, when feature perturbations result in the spatial overlap of distinctive features from different letters, both identification and relative location errors are assumed to occur, and thus the observed correlation between relative location and identification accuracy can be accounted for in terms of the model.

\section{REFERENCES}

ARABIE, P. Perception of letter strings as a function of constituent letter presentations. Unpublished doctoral dissertation, Stanford University. 1974.

Bouma, H. Interaction effects in parafoveal letter recognition. Nature, 1970, 226, 177-178.

Bouma, H. Visual interference in the parafoveal recognition of initial and final letters of words. Vision Research, 1973, 13, 767-782.

ERIKsen, C. W., \& ERIKsen, B. A. Visual perceptual processing rates and backward and forward masking. Journal of Experimental Psychology, 1971, 89, 306-313.

Estes. W. K. Interactions of signal and background variables in visual processing. Perception \& Psychophysics, 1972, 12, 278-286.

Estes, W. K., Allmeyer, D. H., \& Reder, S. M. Serial position functions for letter identification at brief and extended exposure durations. Perception \& Psychophysics, 1976, 19, 1-15.

Estes, W. K., Bjork, E. L., \& SkaAr, E. Detection of letters and letters in words with changing vs. unchanging mask characters. Bulletin of the Psychonomic Society. 1974, 3, 201-203.

GARDNER, G. T. Evidence for independent parallel channels in tachistoscopic perception. Cognitive Psychology, 1973, 4, 130-155.

Krumhansl, C. L., \& Thomas, E. A. C. Extracting identity and location information from briefly presented letter arrays.
Perception \& Psychophysics, 1976, 20, 243-258.

Krumhansl, C. L., \& Thomas, E. A. C. Effect of level of confusability on reporting letters from briefly presented visual displays. Perception \& Psychophysics, 1977, 21, 269.279.

MAYZNER, M. S., \& GREenberg, J. Studies in the processing of sequentially presented inputs with overprinting paradigms. Psychonomic Monograph Supplements, 1971. 4(4, Whole No. 52), $73-84$.

Mayzner. M. S., Tresselt, M. E., Checkes, J., \& Hoenig, A. Visual information processing of sequentially presented inputs: III. Further effects of list length and interstimulus interval values on sub-span storage and retrieval mechanisms. Perception \& Psychophysics, 1970. 7, 294-296.

MCINTYRe, C., Fox, R., \& Neale, J. Effects of noise similarity and redundancy on the information processed from brief visual displays. Perception \& Psychophysics, 1970, 7, 328-332.

SHAw, P. Processing of tachistoscopic displays with controlled order of characters and spaces. Perception \& Psychophysics. 1969. 6. 257-266.

ShIFFrin, R. M., \& GaRDNeR, G. T. Visual processing capacity and attentional control. Journal of Experimental Psychology. $1972,93,72-82$

Sperling, G. A model for visual memory tasks. Human Factors, 1963. 5, 19-31.

TOWNSEND, J. T. Theoretical analysis of an alphabetic confusion matrix. Perception \& Psychophysics, 1971, 9, 40-50.

Wolford, G. Perturbation model for letter identification. Psychological Review, 1975, 82, 184-199.

Wolford, G., \& Hollingsworth, S. Lateral masking in visual in tormation processing. Perception \& Psychophysics, 1974, 16, $315-320$.

\section{NOTES}

1. A detailed account of the assumptions of the model, analytic expressions for the probability of correct identification, and the estimated parameter values are available from the author upon request.

2. Also available from the author is a detailed description of the assumptions of the location model and a demonstration that the model is able to account for the effects of mode of presentation on absolute and relative location accuracy.

(Received for publication February 22, 1977; revision accepted July $11,1977$. 